\title{
Coordinated Signal Control System in Urban Road Network
}

\author{
https://doi.org/10.3991/ijoe.v16i10.15473 \\ Nouha Rida $\left.{ }^{(}\right)$ \\ (RIME), Mohammed V University, Rabat, Morocco \\ nouharida@gmail.com \\ Mohammed Ouadoud \\ Abdelmalek Essaâdi University, Tetouan, Morocco \\ Abderrahim Hasbi \\ (RIME), Mohammed V University, Rabat, Morocco
}

\begin{abstract}
Road congestion has become one of the major problems of urban cities due to the growth in the number of vehicles and the limitation of road infrastructure. The consequences of this congestion can be seen through a difficult movement of vehicles, an increase in $\mathrm{CO} 2$ emissions, increasing energy consumption, and stress for drivers. Intelligent Transport Systems (ITS) based on wireless sensor networks (WSN) have emerged with new solutions and application potentials in the context of the Intelligent city.In this article, we study an approach of using a wireless sensor network to control the traffic lights of several intersections and optimize their performance by reducing the average waiting time of users. the proposed adaptive traffic light controller determines the traffic lights sequence in real-time based on the current traffic situation at the local intersection, the expected arrivals, and the degree of congestion at destination intersections. To evaluate our approach, we construct an intersection network of twenty-four intersections with high traffic intensity using the SUMO simulator, The results of the simulation demonstrate the efficiency and practicality of the proposed algorithm.
\end{abstract}

Keywords - Coordinated traffic light control, intelligent transportation system, traffic light control, wireless sensor network for smart mobility.

\section{Introduction}

The traffic control system allows managing traffic flows, improving the safety of vehicles and pedestrians on the roads, and facilitating the use of the road network. It is materialized through measurement and detection systems (video, radar, loop), traffic light-signaling equipment (traffic lights, sound devices), connection and coordination devices counting stations, panels with variable messages, and road sensors.

Among these different means of action, traffic lights play an important role in ensuring safety by sharing over time the use of the same space between conflicting flows. 
However, by choosing the duration of each color (green,red) and synchronizing the traffic lights between them, they also make it possible to manage the flow of the demand. Different methods and a multitude of tools exist to ensure this management by traffic lights. The predetermined traffic lights plan, which is based on time scheduling, forms the basis of the management system of the vast majority of cities in the world. To this basic brick, methods have been added to make it possible to ensure the management system-based traffic lights plan adaptation for significant traffic and maintain real efficiency in wide operating ranges, as long as system monitoring and maintenance are ensured.

Nevertheless, over the last twenty years, a new family of the traffic control so-called "real-time" or "adaptive" systems have emerged, which tends to stand out from the traditional traffic lights plans. It offers more flexibility in the choice of traffic light conditions and allows adaptation to the evolution of traffic over the years without requiring updating as in the case of fixed traffic lights plans.

This family actually gathers systems that are extremely different from one to another with different methods and specificities. Most of these systems consider only the traffic status and the information that precedes the intersection in question. On the other hand, others are cooperating; they connect the various intersections of the network with each other to make the exchange of information between neighboring intersections possible. Sharing this information makes it possible to optimize the traffic according to its state at the local intersection and its neighbors.

In this work, we propose a traffic light control system for adjacent intersections that cooperate and adopt a traffic light policy.

The paper is organized as follows: after the introduction, we present the problem and context in the second section. In the third section, we discussed related literature and presents the system network. While in the fourth one, we propose a wireless sensor network topology for traffic monitoring that ensures the availability of traffic status for a network of road intersections. The fifth section presents our coordinated traffic light control algorithm to determine the sequence of phases and the time of green light according to the traffic on the intersections network. Finally, in the sixth section, we evaluate the proposed method via the SUMO simulator, and we demonstrate its effectiveness by comparing the results to other approaches.

\section{$2 \quad$ Related Works}

As mentioned in the previous section, there are two main types of traffic control management. There is a fixed time controller, with a predetermined and fixed cycle, and there is also an adaptive controller that modifies the cycle sequence and the duration of the phases as a function of the numbers of the vehicles present on each lane.

In [1], the authors propose an adaptive controller based on the theory of the queue and the number of vehicles in each direction as a decision criterion. However, the controller treated in [2] is based on a dynamic fluid model. The system proposed in [3] detects the level of congestion and abnormal situations in two main highways and for 
four intersections. It makes a real-time decision to determine the green-light interval for each traffic light at each intersection based on the genetic algorithm.

The self-organization of traffic lights based on historical traffic status data is presented in $[4,12]$. The controller in [5] is cooperative between a network of intersections and semi adaptive.

In another study [6], the authors proposed a multi-objective scheme ant colony optimization algorithm for a traffic light (ASTL) to control dynamically the cycles of traffic lights. This solution controls the traffic light by using a nature-inspired solution called ant colony optimization.

The method proposed in [17] selects the sequence of phases composing a cycle according to several criteria which are the presence of priority vehicles, the duration of the periods without detection of new arrivals, the causes of famine, the total time of waiting and the length of the queues. In [20] and [21], we inspired by the "Smallest job first" method of scheduling tasks to run them by a computer processor. We proposed the "Smallest phase first" algorithm which gives priority to phases with the smallest queues. Subsequently, in [22], we integrated the waiting time in the algorithm proposed in [21].

In $[16,31,36-39]$, the authors find that the traffic road control problem at the intersections is a multi-agent system, in which each agent adjusts the traffic lights according to the traffic variations in real-time.

For intersections control, the main difference between the proposed multi-agent systems models in the literature exists in their agent design approaches. They choose to classify different entities as agents, such as an intersection, the traffic light controller, or a vehicle.

To adapt traffic lights to traffic changes, researchers use reinforcement learning (RL) methods [19,41-46]. Traditional reinforcement learning is difficult to use and implement due to two main challenges, the first is the presentation models of the environment, the second is the modeling of the link between intersections, and the third is the decision and the environment. To meet these challenges, researchers use deep reinforcement learning techniques, such as Q-Learning and Deep Q-Learning [40, 42, 46].

Problems using reinforcement learning techniques are modeled in most cases as a Markov decision process which chooses its decision according to three factors that are the system state (S), the action (A), and the (R) reward function. In the literature, there are several works based on RL, which propose strategies, that provide a state-to-action mapping to choose the most cost-effective action for a given state. The goal of those works is to find the optimal action with the greatest overall reward. These approaches are generally distinguished by the choice of the three factors listed below. For example, for the state, some choose the number of vehicles in the queues, or the waiting time, etc. Using various reward functions can be mono-objective or multi-objective.

Neural networks are inspired by the functioning of biological neurons and implement learning by experience. In the case of road traffic, several authors have studied this scheme (examples: [14]). Here, it is a question of a quick classification and learning to improve them, rather than going through traditional modeling. 
In [7], the authors propose a real-time traffic monitoring system that combines a wireless sensor network with several fuzzy logic controllers. For each phase, the authors use a fuzzy logic controller to determine the duration of its green light.

To solve the problem of traffic light synchronization, in [32, 43], the authors use a centralized approach. The main objective of the optimization method, proposed in the first work, is to minimize the total time delay of the network in a given finite horizon, while in the second one, is to optimize the vehicle flow in a fixed lapse of time. Similarly, the signaling control method proposed in [29] aims at minimizing queue lengths and maximizing throughout. In [34], Relative backlog rate-based signaling has been proposed. The algorithm proposed in [27] is based on the delay to optimize the flow of vehicles crossing the intersection during the green light. In [30], the authors proposed two methods, the Minimum Destination Distance First (MDDF) and the First Minimum Average Destination Distance (MADDF) to maximize the flow of traffic.

In [28], the authors propose a traffic light management algorithm according to the real-time distribution of vehicle flows around the intersection. The proposed algorithm also allows the management of the emergency vehicle passage by defining the sequence of phases in such a lane as to allow the emergency vehicles approaching the intersection to go green without stopping.

In [18], the authors are interested in a network covering several intersections, and they extend their proposed solution in [23] to fit an intersection network. They proposed a distributed algorithm that allows sensors to constantly cooperate and adapt the traffic lights strategy to traffic conditions. To simplify and make their algorithm effective, they define a score of three criteria to be used at several intersections to evaluate each movement of an intersection and to select the most important ones according to the vehicles presented in all intersections.

The traffic information used in these strategies is collected by inductive loops [11], cameras [6,13], radars, VANET (ad hoc network for vehicles) [15], or a wireless sensors network $[1,17,18]$.

For a controller based on a wireless sensor network, the topologies proposed in the literature in $[1,10,118]$ are composed of a sensor or two per direction to specify the number of vehicles in the queue. In [24], the authors studied the influence of the choice of topology with a sensor per direction and two sensors per direction on the average waiting time. They found that a topology with two sensors gives a reduced vehicle waiting time compared to that with a sensor per direction.

\section{Problem and Context}

The constriction of the traffic light plans, cycles, phase sequences, green light duration, and the traffic light synchronization between adjacent intersections are the basis of the traffic control system.

In cities, traffic light control systems are generally of two types: static plan control and adaptive control, that react in real-time to the traffic variations. The first type of regulation allows the same sequence of phases to be repeated indefinitely with the same duration of the green light, it is less expensive and older, while the second is more 
efficient and complex. It consists of determining the sequence of green lights according to the actual traffic situation at a single intersection, for an isolated strategy or coordinated strategy in a network of intersections. For normal cases and in the presence of emergency vehicles or accidents.

These adaptive systems respond to the changes in road traffic to achieve the following objectives: maximizing traffic flow through intersections during the green light, improving road safety, minimizing vehicles waiting times at intersections, reducing energy consumption and reducing the number of stops that vehicles must make during their journeys on the urban network.

Our solution allows adaptive control of road traffic in a network of adjacent intersections. An intersection among these intersections presented in figure 2 consists mainly of four directions (N, S, E, W), with three movements for each: go straight, turn left and turn right (figure 1). Turning right will not be considered because vehicles are always allowed to turn right.

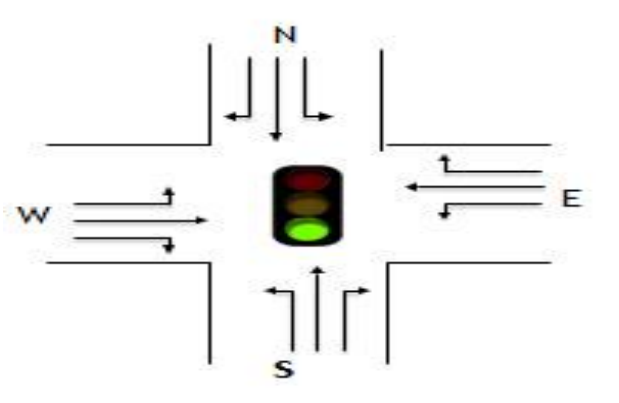

Fig. 1. The studied intersection model

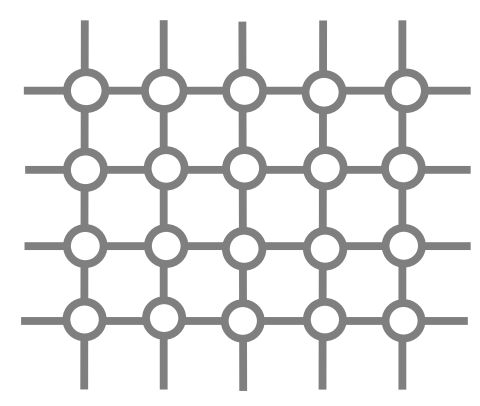

Fig. 2. A $6 \times 4$ network of traffic intersections

Each movement of the intersection is represented by a symbol composed of a combination of two characters. Hence, the two characters represent the cardinal directions ( $\mathrm{N}$ for the north, $\mathrm{S}$ for the south, $\mathrm{W}$ for the west, and $\mathrm{E}$ for east) for the source destination. For example, in figure 1, direction W includes movements: WE, WS, and WN.

A traffic phase consists of a set of movements that allows waiting vehicles to cross the intersection at the same time without conflict. With the model used in this work, we have eight possible phases that are presented in figure 3 .

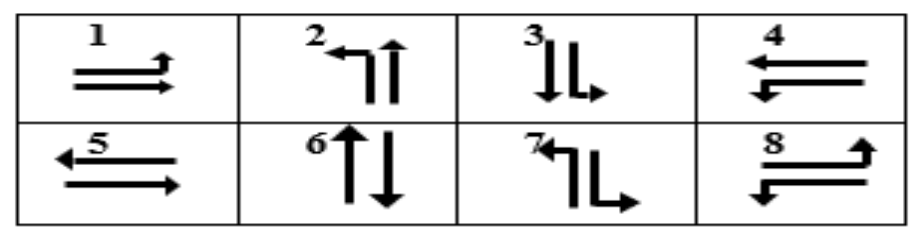

Fig. 3. The possible phases for the studied intersection model. 


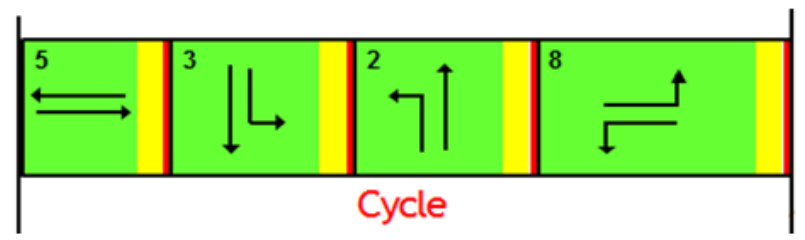

Fig. 4. Example of a traffic light cycle.

Considering a network of connected intersections, the main idea of our coordinated system is to design the phases of a traffic light cycle in real-time, according to the traffic conditions at the intersection concerned and its neighboring intersections.

- The proposed solution consists of three parts, which are illustrated in figure 5: The first part is devoted to presenting the traffic monitoring system based on a wireless sensor network.

- A second part is meant to determine the sequence of the phases in which we specify the criteria and the process of choice of the next phase. And the last one is about calculating the duration of the green light according to the vehicle number of the phase chosen in the second part.

Below, we will explain in detail each one of these parts.

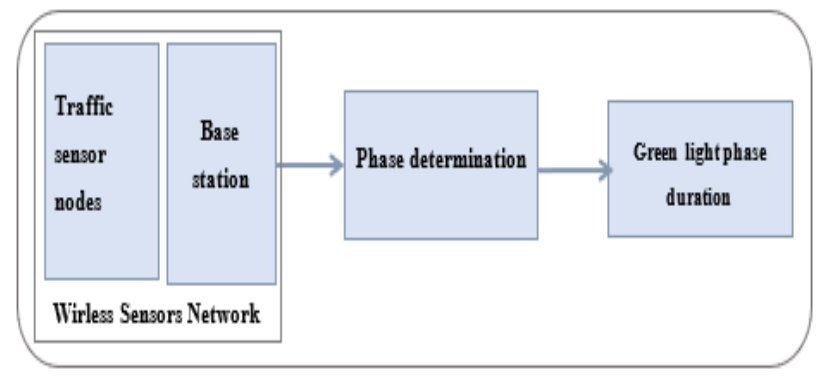

Fig. 5. Chart showing the data flow for traffic light controlling

\section{$4 \quad$ Network Topology and Data Collection}

Traffic knowledge in real-time is an essential element for the operation of the road network and in particular to feed the traffic management systems. A wireless sensor network is responsible for collecting traffic data such as queue lengths and waiting times in each road.

The traffic monitoring system used in this document consists of several magnetic sensor nodes installed in the middle of the road. These sensors are used to detect and classify vehicles through evaluating the distortion of the Earth's magnetic field produced by the presence of ferrous objects [26]. It is noteworthy to mention that very few contributions have proposed realistic models for deploying wireless sensor networks in 
an urban network of intersections as part of an intelligent transport system and the smart city.

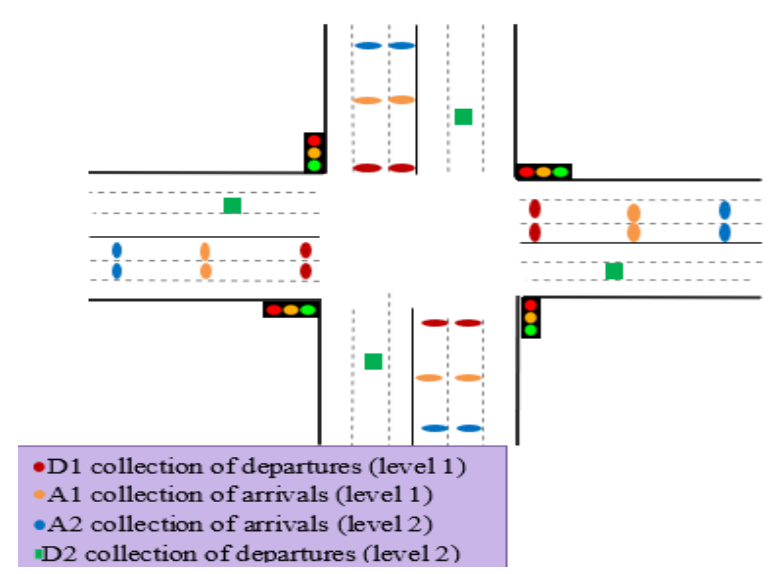

Fig. 6. WSN topology for an intersection

In figure 6, we have shown the proposed topology to control the traffic lights in real-time. The topology consists of four nodes per direction, that two of which count the number of arrivals and the others count the number of departures.

In figure 6, we have presented the wireless sensor network topology used to monitor the traffic at an intersection. It consists of four nodes per direction, two of which count the number of arrivals and the others specify the number of departures. Next, we will explain the role of each sensor in our topology and the reason behind choosing a topology with four sensors per direction and not only two sensors as the solutions proposed in $[1,7,10,18]$.

- Node D1: Allows detecting the number of departures, i.e. the number of vehicles that have left the queue to cross the intersection.

- Nodes A1 and A2: count the number of arrivals. The events detected by these two sensors and those with sensor D1 allow the queue to be calculated and classified the traffic flow into two classes: a normal class for a queue located between node D1 and A1, and an important class for a queue that goes from node A1 to node A2.

- Node D2: Specifies the number of vehicles leaving the local interaction to the nearby intersection. The information collected by this sensor will be transmitted to the next intersection on the same lane.

In addition to the other information specified by a topology with two sensors per direction which are waiting time and queue lengths, with a topology of four nodes per lane, it is possible to classify the flow and determine the number of vehicles leaving the intersection to another, so that the latter can predict the number of arrivals.

The distance between sensors on the same input path depends on the number of vehicles that define the size of these flow classes. For a normal class, the distance between sensors D1 and A1 is calculated according to the number of vehicles defining this class 
multiplied by the average length of the vehicles, which is 6 meters. The distance between the A1 and A2 sensors can be defined, for example, as a distance capable of detecting a queue of 25 vehicles. These values can be adjusted according to the specification of the number of vehicles that define each flow class.

The events captured by these sensors will be communicated to a decision node via their wireless interfaces. This node is responsible for collecting traffic data from the local intersection and traffic data sent from nearby intersections to produce a traffic signal management decision. It also allows traffic data to be shared with other nearby intersections via wired or wireless communication.

\section{$5 \quad$ Next Phase Determination}

In the case of a coordinated system for adjacent intersections, it is necessary to predict the number of arrivals at an intersection at any time using the number of vehicles that have left the adjacent intersections towards that intersection.

Since it is very difficult to instantly assess the exact traffic situation at all intersections due to its very dynamic environment, we worked with the following assumptions:

- The travel time of a vehicle between two adjacent intersections has a constant value. The travel is expressed according to the following equation:

$$
\text { Travel }=V_{\text {avg }} / d
$$

By "Travel" means the travel time, Varg is the average speed of the vehicle between the two intersections that are estimated constant, and $\mathrm{d}$ is the distance between the two intersections.

- Flow conservation, i.e. the number of vehicles entering one roadway front is the same number of vehicles leaving the other roadway front.

Our proposed solution, in this work, consists of designing the traffic light cycle phases according to the traffic conditions at the local intersection concerned by this control, and at the adjacent intersections (figure 7). The wireless sensor network presented in the previous section collects traffic information in different directions, they are in the operation of this algorithm. 


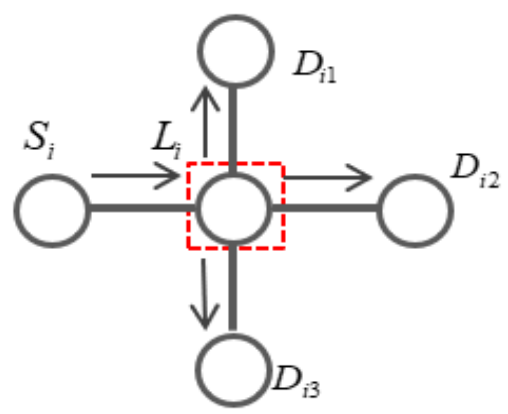

Fig. 7. Example of Interactions Between Intersections: local (Li), source Si and destinations (Di1, Di2, and Di3 )

In figure 7, we show an example of the interactions between the intersection at the center of $\mathrm{Li}$ and its neighbors, and for the movements (WS, WE, WN). The intersection Si represents the source of vehicles that arrives at these movements from the intersection Li. Di1, Di2, and Di3 are the intersections destination of the vehicles coming from Li. Arrows in the figure indicate the traffic flow between these intersections.

To introduce the influence of traffic conditions at each of these intersections in the choice of the next green light at $\mathrm{Li}$, we defined three factors for each movement at this intersection.

\subsection{Factors}

F1 factor: This is an indicator of traffic conditions at the local intersection. It allows privileging movements with large queues in the choice of the next phase that will have the green light. This indicator is calculated according to the following two equations.

$$
\begin{aligned}
& F_{1}=\frac{Q_{j}}{\sum_{0}^{11} Q_{k}} \\
& Q_{j}(t)=A R_{j}(t)+A G_{j}(t)+Q_{j}(t-1)-D P_{j}(t)
\end{aligned}
$$

Where:

$\sum_{0}^{11} Q_{k}$ : is the number of vehicles in all the movements of the intersection.

$\mathrm{AGj}$ : The vehicle's number arriving during a green light and for the lane $\mathrm{j}$.

ARj: The vehicle's number arriving during the red light and for the lane $\mathrm{j}$.

$Q_{j}(t)$ : The queue length for lane $\mathrm{j}$ and cycle $\mathrm{t}$.

$Q_{j}(t-1)$ : The vehicle numbers that remained from the last green light.

$\mathrm{DPj}$ : The departures number during a current green light. 
F2 factor: At the beginning of each phase, the controller must predict the number of vehicles that will arrive at the intersection before the end of the next phase. The role of this factor is to minimize the number of stops at intersections, synchronize traffic lights between intersections and create green waves. To calculate it, it is first necessary to determine the number of vehicles that have already left the intersection $\mathrm{Si}$ to $\mathrm{Li}$ and trying to reach Li before the end of the next phase that has a green light duration required for the waiting queue calculated by the factor F1.

To specify the value of F2, the controller uses the data captured by sensor D2 at the intersection Si.

In the following figure 8 , we show the time variations in the movements of the two vehicles v1 and v2 between the intersections $\mathrm{Si}$ and $\mathrm{Li}$.

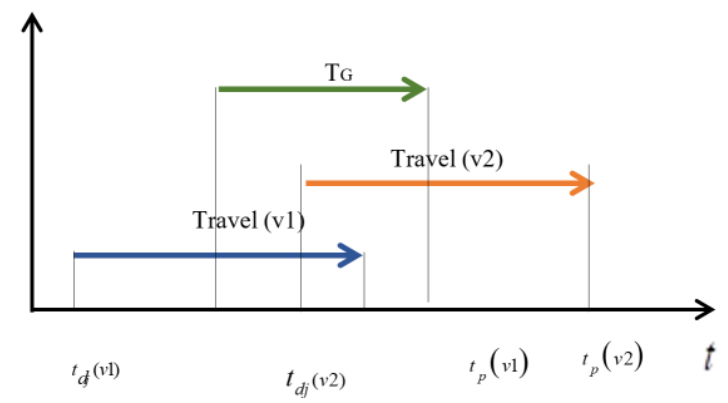

Fig. 8. Travel of two vehicles v1 and $v 2$ between two intersections as a function of time.

Any vehicle verifies the following inequality shall be considered in the factor $F_{2}$.

$$
t_{p} \leq t_{d j}+\text { Travel }<t_{p}+G T
$$

With, tp is the starting time of the next phase, and tdj is the moment when the vehicle $\mathrm{j}$ left the last intersection. Travel is the travel time between the two intersections.

The green time for the next phase GT is estimated according to the following equation:

$$
G T=4+2 * Q_{j}
$$

$\mathrm{Q}_{j}$ is the length of the largest queue of movements in the next phase.

Provided that $\mathrm{N}_{\mathrm{j}}$ is the number of expected arrivals during the phase, the movement $\mathrm{j}$ and $\sum_{0}^{11} N_{k}$ are the numbers planned for the entire intersection. F2 is given as follows:

$$
F_{2}=\frac{N_{j}}{\sum_{0}^{11} N_{k}}
$$


F3 factor: This third factor indicates the congestion degree at destination intersections. It allows the controller to focus on movements leading to slightly loaded intersections rather than congested intersections.

$$
F_{3}=\frac{1}{Q_{j}} \text { if } Q_{j} \neq 0 \text { else } F_{3}=1
$$

$\mathrm{Qj}$ is the number of vehicles at the destination intersection, and precisely in the lane connecting the local intersection and the destination intersection ( $\mathrm{Li}$ to $\mathrm{Di}$ in figure 7).

After calculating the three factors for each movement, we determine them for all phases of the intersection concerned by this control and which are illustrated in figure 4.

For a phase $\mathrm{P}$ composed of two movements (M1, M2), the three factors (F1, F2, F3) are calculated as follows:

$$
\begin{aligned}
& F_{1}(P)=\frac{F_{1}(M 1)+F_{1}(M 2)}{2} \\
& F_{2}(P)=\frac{F_{2}(M 1)+F_{2}(M 2)}{2} \\
& F_{3}(P)=\frac{F_{3}(M 1)+F_{3}(M 2)}{2}
\end{aligned}
$$

For each phase $\mathrm{P}$, a score $\mathrm{S}(\mathrm{P})$ will be defined as the weighted sum of these three factors, according to the following equation.

$$
S(P)=\frac{\alpha F_{1}(P)+\beta F_{2}(P)+\gamma F_{3}(P)}{\alpha+\beta+\gamma}
$$

$\alpha, \beta$ and $\gamma$ are three weights that can be used to give complementary importance to each of these factors.

\subsection{Selection procedure of the next phase}

Finally, at the end of each phase, the controller chooses the phase that will be given the green light according to the following rules:

- Case 1: If traffic flow class at the local intersection is of the important class(F1>Icongestion the degree of congestion), then only the congestion problem at that intersection is addressed. Thus, the next phase will be the phase with the largest $\mathrm{F} 1$, i.e. the phase with the largest queue.

- Case 2: If the first case is not achieved, we start at the third factor F3 to determine the degree of congestion at the destination intersections. 
- If F3 exceeds a threshold that defines the critical state of congestion(F3>Icritical), then all directions from the local intersection to this congested intersection will be eliminated from the next phase selection and the third case will follow.

- Case 3: If the first case is not realized, then the next phase will be the phase with the maximum score defined in equation 11.

- Case 4: For an isolated intersection that has no F2 and F3, the decision is based only on the first factor F1, from which the next phase will be the phase with the largest F1.

\subsection{Light length determination}

In this work, the cycle represents only a succession of phases that compose all the possible movements. Unlike most strategies, there is no explicit concept of a cycle. The phases follow one another, and COTLA ensures it all.

Once the movements are selected, in line with the criteria outlined in the previous sections, the controller calculates the green light time of the phase according to equation 11.

TG is calculated based on the load of the track with the largest number of vehicles. This leaves the possibility of being able to empty all the concerned lanes at the intersection.

$$
T_{G}=\max \left(T_{S}+T_{h} * Q_{L}, T_{\max }\right)
$$

TG is delimited by a minimum time and a maximum time Tmax. A phase that is too short may not leave enough time for a queue to unload. Conversely, a phase that is too long increases the waiting time for the drivers present on the other phases, without this being useful.

In some systems, this time is calculated dynamically and ranges from a minimum time to a maximum time $[33,35]$.

\section{Simulation and Evaluation}

To evaluate the performance of our approach, accurate information on vehicles traveling at an intersection is necessary. Ideally, our model should have been tested on existing crossroads and compared its performance against the solutions discussed in the literature review. Unfortunately, we do not have access to such intersections, and we do not have enough information on any models to replicate them and compare their results with ours. Therefore, we used the SUMO simulator [25], which models the behavior of vehicles at the various crossroads flows. This simulator allows us to reproduce user behavior by generating observations that would normally come from the detectors.

Our simulation tool allows determining a set of information about each vehicle, such as: waiting for time, position, fuel consumption, $\mathrm{CO} 2$ emission, etc. The behavior of vehicles varies according to the type of flow to which they belong (turns left, turns right and goes straight), their initial position, and the condition of the traffic light. 
We tested our approach in a network of intersections, $6 \times 4$ intersections (figure 9), separated by a distance of $1 \mathrm{~km}$.

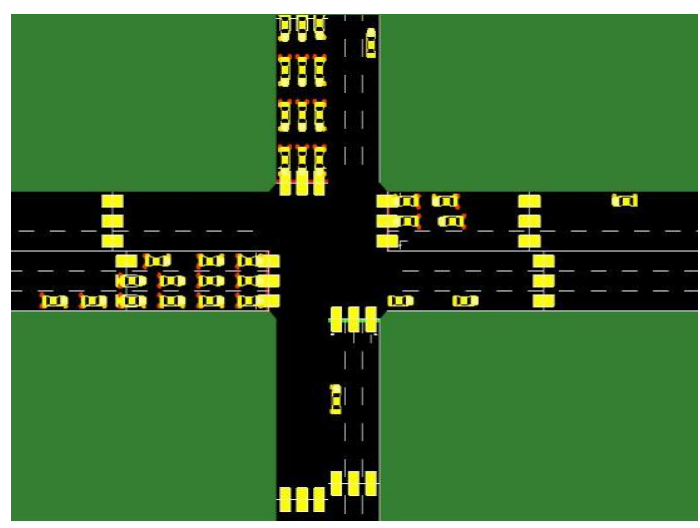

Fig. 9. The controlled intersection in SUMO

The simulations are performed for 1300 seconds, and 3600 vehicles enter intersections according to a constant fish distribution between 0.1 and 0.05 .

As in reality, vehicles are considered to enter the coverage area of a crossroads and a network of adjacent crossroads continuously, the simulation of continuous flows must be performed to test the influence of the proposed algorithms on traffic. The four control criteria (average waiting time at an intersection, average queue length, fuel consumption, and $\mathrm{CO} 2$ emission) will be studied separately to test the influence of our traffic control approach on these criteria.

Finally, we will also compare the simulation results of our approach with some existing regulation strategy results that are:

- A fixed time controller with four phases of 25 - or 20 -seconds duration and which is illustrated below:

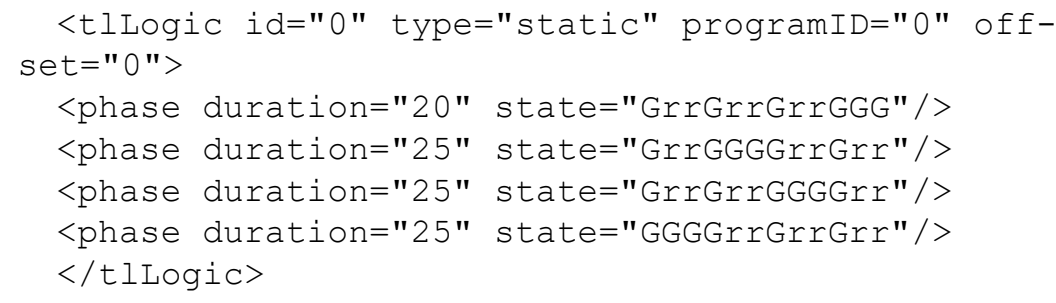

- The TSTMA algorithm proposed in [1], which prioritizes the lanes with the largest queue (isolated strategy).

- The SPF method, which uses the smallest queue (isolated strategy) as the criterion for choosing the next phase. 
- Adaptive TOPIOCA algorithm for connecting intersections, it is proposed in [18] (coordinated strategy).

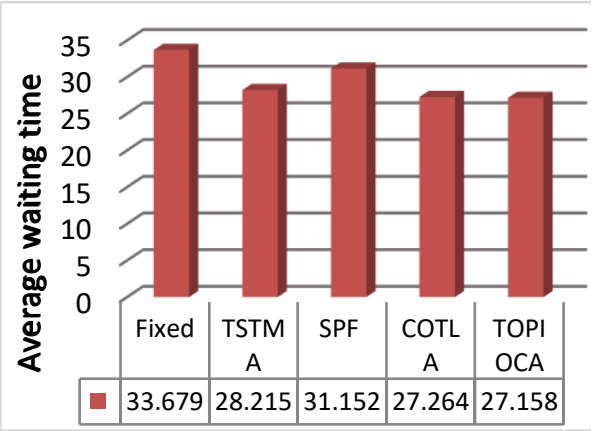

Fig. 10.The average waiting time comparison between the four controllers

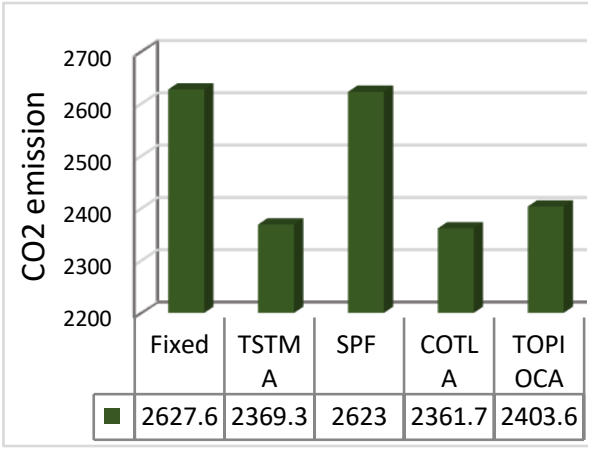

Fig. 12.The $\mathrm{CO} 2$ emission $(\mathrm{kg})$ comparison between the four controllers

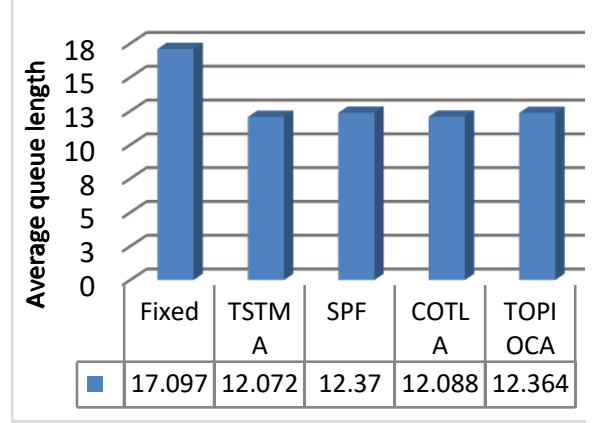

Fig. 11.Average queue length: comparison between the four controllers

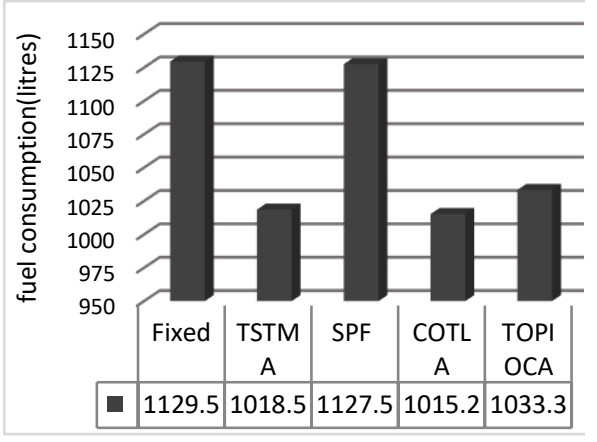

Fig. 13. The fuel consumption in liters: Comparison between the four controllers

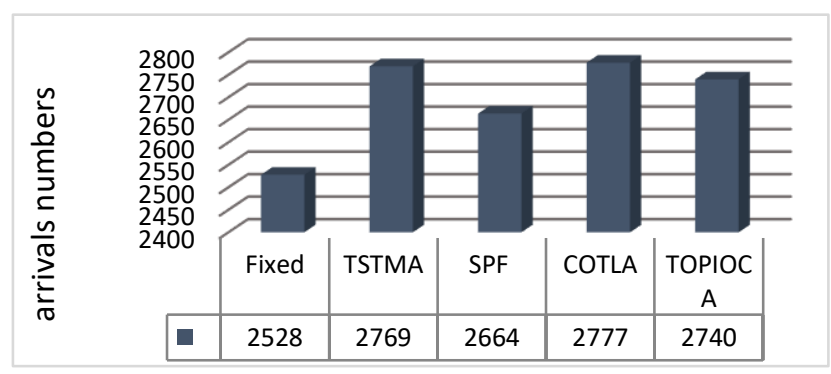

Fig. 14. The arrivals numbers: comparison between the four controllers. 
Figure 10 shows the average waiting time for each vehicle in the simulated road network and according to the five methods used. Our approach is working best. Our algorithm can reduce the average waiting time to $33 \%, 11 \%, 23 \%$, and $7 \%$ respectively for static controller, ASTMA, SPF and TOPIOCA algorithms.

While the algorithm achieves the best results by reducing the average queue length (figure 11). However, the reduction rate is very low compared to the one obtained by our COTLA algorithm, which is $0.1 \%$. Our algorithm has a reduced rate of $41 \%$ in the average queue length compared to the static controller and $2 \%$ compared to SPF and TOPIOCA.

Figure 14 shows the number of vehicles arriving at their destinations during the simulation time and with the five methods. The results show that the largest numbers of arrivals are obtained by our COTLA method followed by the TSTMA and TOPIOCA methods. This means that a road network with traffic controllers using our COTLA algorithm is less loaded than a traffic network with one of the other controllers.

Figure 12-13 respectively represents the volume of the fuel consumption and the amount emitted in $\mathrm{CO} 2$ by the vehicles in the simulation. The results show that COTA and TSTMA give the lowest fuel consumption and $\mathrm{CO} 2$ emission with the same reduced rates for both: $10 \%$ compared to the fixed-time controls, $17 \%$ for the TOPIOCCA algorithm, and $1 \%$ compared to the SPF.

In summary, based on these results, our coordinated algorithm for a network of connected intersections reduces network load, waiting times, and energy consumption as well as improves traffic flow. Moreover, $\mathrm{CO} 2$ emissions are better than adaptive controllers in isolated intersections or fixed-time controllers. Indeed, SPF and TSTMA are two adaptive algorithms that exploit certain parameters to optimize one or two objectives, such as the average waiting time of each vehicle and the length of the queue in each lane. Their weakness is that they change the traffic based on the data measured at the intersection, and they do not try to predict the entry of new vehicles at the intersections. COTLA and TOPIOCA, however, manage the vehicle flow sequence in the intersection using its surveillance system (the first-level sensors installed at the intersection concerned by the traffic signal control), and the data from neighboring intersection sensors, which will be used to predict traffic status for a given time and for synchronizing the traffic lights.

\section{Conclusion}

In this article, we have proposed an adaptive traffic light control approach for multiple intersections using a wireless sensor network. The traffic light control algorithm allows traffic light controllers to cooperate and adapt the control policy to traffic conditions at multiple intersections. In addition to adjusting the traffic lights to the traffic conditions at the intersection concerned by this control, the algorithm predicts the number of arriving vehicles at the intersection during the next phase and calculates the degree of congestion for the intersection's destinations. Our approach takes into consideration traffic conditions in nearby intersections to favor less dense intersections than 
the most congested ones, and to decrease the average waiting time and the number of drivers' stops.

Using the SUMO traffic simulator, we simulate the traffic on a network of twentyfour intersections.

The simulation results obtained by our algorithm are better than those obtained by a fixed time control or control strategies for isolated intersections. The results demonstrate the efficiency and practicality of our approach to reduce average vehicle waiting times and queue lengths, thereby reducing road network load and improving traffic flow.

In future work, we will build networks based on several intersection models with different road traffic scenarios to assess the performance of our approach in different scenarios. Also, we will equip the traffic monitoring system with the ability to detect emergencies (such as the presence of ambulances, trucks, public transportation, etc.) using less expensive sensors; implementing appropriate emergency measures to rank priorities.

\section{$8 \quad$ References}

[1] Yousef, Khalil M., Mamal N. Al-Karaki, and Ali M. Shatnawi., Intelligent traffic light flow control system using wireless sensors networks, J. Inf. Sci. Eng, vol 26(3): 753-768, 2010.

[2] Helbing, Dirk, Stefan Lämmer, and Jean-Patrick Lebacque, Self-organized control of irregular or perturbed network traffic (Springer, Boston, MA, 2005, pp. 239-274). https://doi.org/ $\underline{10.1007 / 0-387-25805-1 \_15}$

[3] Odeh, Suhail M., Management of an intelligent traffic light system by using genetic algorithm, Journal of Image and Graphics, vol. 1 (2): 90-93, 2013. https://doi.org/10.12720/joig $.1 .2 .90-93$

[4] Burguillo-Ril, Juan C., et al. "History-based self-organizing traffic lights." Computing and Informatics 28.2 (2): 157-168,2012.

[5] ZHU, Qin, PENG, Chao, SHI, Jingmin, et al. Cooperative traffic light control based on semireal-time processing. Journal of Automation and Control Engineering, vol. 4 (1) : 40-46, February 2016. https://doi.org/10.12720/joace.4.1.40-46

[6] Rida, N., Ouadoud, M., \& Hasbi, A. (2020). Ant colony optimization for real time traffic lights control on a single intersection. International Journal of Interactive Mobile Technologies (iJIM), 14(02), 196-214. https://doi.org/10.3991/ijim.v14i02.10332

[7] GARG, Hiteshi et KAUSHAL, Er Gautam. Traffic Lights Control System for Indian Cities Using WSN and Fuzzy Control. Traffic, vol. 4(7) : 2587- 2592, 2017

[8] Collotta, Mario, Lucia Lo Bello, and Giovanni Pau, A novel approach for dynamic traffic lights management based on Wireless Sensor Networks and multiple fuzzy logic controllers, Expert Systems with Applications, vol. 42(13): 5403-5415, 2015. https://doi.org/10.1016/j.e swa.2015.02.011

[9] Salman, Muntaser, Suat Ozdemir, and Fatih Celebi., Fuzzy traffic control with vehicle-toeverything communication,Sensors, vol.18(2):368,2018.https://doi.org/10.3390/s180203 $\underline{68}$

[10] Zou, F., Yang, B., \& Cao, Y., Traffic light control for a single intersection based on wireless sensor network, 9th International Conference on Electronic Measurement \& Instruments, pp. 1040-1-1044, Beijing, China, August 2009. https://doi.org/10.1109/ICEMI.2009.5273 $\underline{994}$ 
[11] Zhu, Y., Liu, X., Li, M., \& Zhang, Q. Pova: Traffic light sensing with probe vehicles, IEEE Transactions on Parallel and Distributed Systems, vol.24(7) : 1390-1400, 2012. https://doi. org/10.1109/TPDS.2012.233

[12] Yousef, Khalil M. Ahmad, Ali Shatnawi, and Mohammad Latayfeh., Intelligent traffic light scheduling technique using calendar-based history information, Future Generation Computer Systems, vol.91:124-135, February 2019. https://doi.org/10.1109/TPDS.2012.233

[13] Odeh, Suhail M., Management of an intelligent traffic light system by using genetic algorithm,Journal of Image and Graphics, vol. 1(2):90-93, 2013. https://doi.org/10.12720/joig.1 $.2 .90-93$

[14] Spall, J. C., \& Chin, D. C., Traffic-responsive signal timing for system-wide traffic control. Transportation Research Part C: Emerging Technologies, vol.5(3-4): 153-163, 1997. https://doi.org/10.1016/S0968-090X(97)00012-0

[15] Khanafer, M., Guennoun, M., \& Mouftah, H. T, WSN architectures for intelligent transportation systems, 3rd International Conference on New Technologies, Mobility and Security, pp. 1-8, December 2009. https://doi.org/10.1109/NTMS.2009.5384685

[16] Tan, M. K., Chuo, H. S. E., Chin, R. K. Y., Yeo, K. B., \& Teo, K. T. K. Hierarchical Multiagent System in Traffic Network Signalization with Improved Genetic Algorithm. IEEE International Conference on Artificial Intelligence in Engineering and Technology (IICAIET), pp. 1-6, November, 2018. https://doi.org/10.1109/IICAIET.2018.8638464

[17] Zhou, B., Cao, J., Zeng, X., \& Wu, H., Adaptive traffic light control in wireless sensor network-based intelligent transportation system, IEEE 72nd Vehicular Technology ConferenceFall, Vol. 72, pp. 1-5, September 2010. https://doi.org/10.1109/VETECF .2010 .5594435

[18] Faye, S., Chaudet, C., \& Demeure, I. A distributed algorithm for multiple intersections adaptive traffic lights control using a wireless sensor networks, the first workshop on Urban networking (pp. 13-18), December 2012. https://doi.org/10.1145/2413236.2413240

[19] Ha-li, P., \& Ke, D., An intersection signal control method based on deep reinforcement learning. 10th International Conference on Intelligent Computation Technology and Automation (ICICTA), pp. 344-348, October 2017. https://doi.org/10.1109/ICICTA.2017.83

[20] Rida, N., \& Hasbi, A. Traffic Lights Control using Wireless Sensors Networks, The 3rd International Conference on Smart City Applications (pp. 14), October 2018. https://doi.org/ $10.1145 / 3286606.3286791$

[21] Rida, N., \& Hasbi, A., Dynamic Traffic Lights Control for Isolated Intersection Based Wireless Sensor Network (Springer, 2018, pp. 1036-1044). https://doi.org/10.1007/978-3-03011196-0_84

[22] Rida, N., Ouadoud, M., Hasbi, A., \& Chebli, S. (2018, October). Adaptive Traffic Light Control System Using Wireless Sensors Networks, IEEE 5th International Congress on Information Science and Technology (pp. 552-556), October 2018. https://doi.org/10.1109/ CIST.2018.8596620

[23] Faye, S., Chaudet, C., \& Demeure, I., A distributed algorithm for adaptive traffic lights control. In 2012 15th International IEEE Conference on Intelligent Transportation Systems (pp. 1572-1577), September 2012. https://doi.org/10.1109/ITSC.2012.6338671

[24] Tubaishat, M., Qi, Q., Shang, Y., \& Shi, H., Wireless sensor-based traffic light control., The 5th IEEE Consumer Communications and Networking Conference (pp. 702-706), January 2008. https://doi.org/10.1109/ccnc08.2007.161

[25] Behrisch, Michael, et al., SUMO-simulation of urban mobility: an overview, The Third International Conference on Advances in System Simulation, pp. 55-60, ThinkMind, 2011.

[26] Jeong, Jaehoon, Wireless sensor networking for intelligent transportation systems, Ph.D. dissertation, Dept. Comp Sci., University of Minnesota., Minneapolis, DEC, 2009.

[27] Yen, C. C., Ghosal, D., Zhang, M., Chuah, C. N., \& Chen, H, Falsified Data Attack on Back pressure-based Traffic Signal Control Algorithms, IEEE Vehicular Networking Conference (VNC) (pp. 1-8), December 2018. https://doi.org/10.1109/VNC.2018.8628334 
[28] Younes, M. B., \& Boukerche, A., An efficient dynamic traffic light scheduling algorithm considering emergency vehicles for intelligent transportation systems. Wireless Networks, vol.24(7) : 2451-2463, 2018. https://doi.org/10.1007/s11276-017-1482-5

[29] Younes, M. B., Boukerche, A., \& Mammeri, A., Context-aware traffic light self-scheduling algorithm for intelligent transportation systems, IEEE Wireless Communications and Networking Conference (pp. 1-6), April 2016. https://doi.org/10.1109/WCNC.2016.7564924

[30] Ahmad, F., Mahmud, S. A., \& Yousaf, F. Z., Shortest processing time scheduling to reduce traffic congestion in dense urban areas. IEEE Transactions on Systems, Man, and Cybernetics: Systems, vol. 47(5): 3900 - 3912, 2016. https://doi.org/10.1109/TSMC.2016. 2521838

[31] Jin, J., \& Ma, X., A Multi-Objective Agent-Based Control Approach with Application in Intelligent Traffic Signal System, IEEE Transactions on Intelligent Transportation Systems. vol. 20(10), 838-855, 2019. https://doi.org/10.1109/TITS.2019.2906260

[32] Kumaran, S. K., Mohapatra, S., Dogra, D. P., Roy, P. P., \& Kim, B. G., Computer Visionguided Intelligent Traffic Signaling for Isolated Intersections, Expert Systems with Applications, vol.134, 267-278, 2019. https://doi.org/10.1016/j.eswa.2019.05.049

[33] KELL, James H. et FULLERTON, Iris J. Manual of traffic signal design (TRB,1991).

[34] SHI, Ming-kang, JIANG, Hong, et LI, Song-huan. An intelligent traffic-flow-based realtime vehicles scheduling algorithm at intersection, The 14th International Conference on Control, Automation, Robotics and Vision (ICARCV), p. 1-5, 2016. https://doi.org/10.1109/ ICARCV.2016.7838779

[35] Yang, Q., \& Koutsopoulos, H. N., A microscopic traffic simulator for evaluation of dynamic traffic management systems. Transportation Research Part C: Emerging Technologies, vol.4(3): 113-129,1996. https://doi.org/10.1016/S0968-090X(96)00006-X

[36] Liu, Y., Liu, L., \& Chen, W. P. (2017, October). Intelligent traffic light control using distributed multi-agent $\mathrm{Q}$ learning, The IEEE 20th International Conference on Intelligent Transportation Systems (ITSC), pp. 1-8, October 2017. https://doi.org/10.1109/ITSC.2017 .8317730

[37] Jin, J., \& Ma, X., Hierarchical multi-agent control of traffic lights based on collective learning, Engineering applications of artificial intelligence, vol.68: 236-248, 2018. https: //doi.org/10.1016/j.engappai.2017.10.013

[38] Belbachir, A.,El Fallah-Seghrouchni, A.,Casals, A.,\& Pasin, M. Smart Mobility Using Multi-Agent System. Procedia Computer Science, vol.151 :447-454, 2019. https://doi.o rg/10.1016/j.procs.2019.04.061

[39] Li, Z., Shahidehpour, M., Bahramirad, S., \& Khodaei, A, Optimizing traffic signal settings in smart cities. IEEE Transactions on Smart Grid, 8(5), 2382-2393, 2016. https://d oi.org/10.1109/TSG.2016.2526032

[40] Wei, H., Zheng, G., Yao, H., \& Li, Z., Intellilight: A reinforcement learning approach for intelligent traffic light control. In Proceedings of the 24th ACM SIGKDD International Conference on Knowledge Discovery \& Data Mining, pp. 2496-2505, July, 2018. https://doi.org/10.1145/3219819.3220096

[41] Ge, H., Song, Y., Wu, C., Ren, J., \& Tan, G., Cooperative Deep Q-Learning With Q-Value Transfer for Multi-Intersection Signal Control. IEEE Access, vol.7 : 40797-40809, 2019. https://doi.org/10.1109/ACCESS.2019.2907618

[42] Van der Pol, E., \& Oliehoek, F. A., Coordinated deep reinforcement learners for traffic light control, Learning, Inference and Control of Multi-Agent Systems, vol.30, pp. 1-8, 2016. https://doi.org/10.1016/j.asoc.2016.07.029

[43] Gao, K., Zhang, Y., Sadollah, A., \& Su, R., Optimizing urban traffic light scheduling problem using harmony search with ensemble of local search. Applied Soft Computing, vol.48: 359-372, 2016. https://doi.org/10.1016/j.asoc.2016.07.029 
[44] Liang, X., Du, X., Wang, G., \& Han, Z., A Deep Reinforcement Learning Network for Traffic Light Cycle Control. IEEE Transactions on Vehicular Technology, vol.68(2), 1243-1253, 2019. https://doi.org/10.1109/PERCOMW.2019.8730706

[45] Zhou, P., Braud, T., Alhilal, A., Hui, P., \& Kangasharju, ERL: Edge based Reinforcement Learning for optimized urban Traffic light control. IEEE International Conference on Pervasive Computing and Communications Workshops (PerCom Workshops), pp. 849-854, March 2019. https://doi.org/10.1109/PERCOMW.2019.8730706

[46] Aslani, M., Seipel, S., \& Wiering, M., Continuous residual reinforcement learning for traffic signal control optimization. Canadian Journal of Civil Engineering, vol.45(8) : 690702,2018. https://doi.org/10.1139/cjce-2017-0408

\section{Authors}

Nouha Rida is currently a Ph.D. Student at Computer Networks, Modeling, and ELearning Research Laboratory at Mohammadia School of Engineer, Mohammed V University, Morocco. She received her degree in engineering in computer science by "Ecole Nationale Superieur de Mine of Rabat".

Mohammed Ouadoud is a Ph.D. in Computer sciences, at the Laboratory of the Information System and Software Engineering (SIGL) at the National School of Applied Sciences, Abdelmalek Essaâdi University. In 2018, he completed his Ph.D. thesis in computer science. His dissertation research focuses on Modeling and Prototyping a New Smart Learning Management System Based on the IMD-LD and the Hybridization between Learning Theories. His current research focuses on E-learning, Software Engineering, Geomatics, and Bigdata. He is a reviewer in several international journals.

Abderrahim Hasbi got his Ph.D. degree in computer science from the University Mohamed 5 of Rabat Morocco. He's a full Professor in Computer Science at the Mohammadia School of Engineering of the University Mohamed 5 Agdal, Morocco. He's a member of the Network and Intelligent systems Group and he has many contributions to researches. nouharida@gmail.com.

Article submitted 2020-05-08. Resubmitted 2020-05-27. Final acceptance 2020-05-28. Final version published as submitted by the authors. 